\title{
latrogenic Hypoglycemia Induced by Valproic Acid in an Adult Patient
}

\begin{abstract}
Literature on antiepileptic-induced iatrogenic hypoglycemia is scanty. Due to its broadspectrum of activity and mechanisms of action, valproic acid (VPA), a fatty acid, is one of the most widely prescribed epilepsy treatments worldwide.

Herein, we describe an adult epileptic patient, in whom persistent, otherwise unexplained, hypoglycemia was most likely induced by VPA, as suggested by the VPA and glucose blood level time course. Indeed, no further hypoglycemic episodes occurred after VPA discontinuation and the diagnostic work-up ruled out other possible causes of hypoglycemia.

This case supports the hypothesis that VPA may induce hypoglycemia, even in the absence of a VPA hepatotoxicity syndrome, due to still not well-defined metabolic mechanisms of action. Moreover, it emphasizes the fact that an iatrogenic pathogenesis should be considered if an apparently unexplained hypoglycemia occurs in a patient on chronic therapy with VPA, even at a therapeutical dosage.
\end{abstract}

Keywords: Hypoglycemia; Valproic Acid (VPA); Epilepsy; Side Effects; Diabetes Mellitus; Iatrogenic Effects; Seizure

CMI 2021; 15(1): 25-29

bttp://dx.doi.org/10.7175/cmi.v15i1.1487

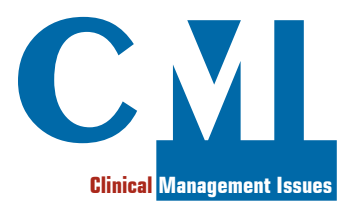

Case Report
1 Neurology Unit, San Giacomo Hospital, Novi Ligure, AL, Italy 2 Internal Medicine Unit, Ovada Hospital, AL, Italy 3 Neurology and Radiology Unit, Guglielmo da Saliceto Hospital, Piacenza, Italy

\section{INTRODUCTION}

Valproic acid (VPA), a branched shortchain fatty acid, is a widely used anti-epileptic drug with a broad spectrum of activity and mechanisms of action [1]. It is used for the treatment of several types of seizure and epileptic syndromes, including generalized and focal seizures [2]. Moreover, it is commonly used for migraine prophylaxis and in the treatment of bipolar disorder, as a mood tone stabilizer. VPA, as a therapeutic agent, is commercially available as Depakote ${ }^{\circledast}$, De-

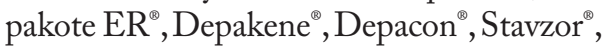
Mylproin ${ }^{\circledR}$, Ergenyl $^{\circledR}$, Dipropylacetic acid, Myproic Acid, and Convulex .

To date, its mechanism of action has not yet been fully clarified. Although VPA does not directly interact with postsynaptic GABA receptors, it does increase regional neuronal GABA concentrations by both inhibiting its metabolism and increasing its synthesis [1].

Preclinical research on animal models has shown the involvement of ion channels, monoamines, the corticotropin-releasing

Why Do we Describe This Case

To the best of our knowledge, very few cases of adult hypoglycemia induced by therapeutical VPA levels have been reported to date. Moreover, this case evidences that the suspicion of an iatrogenic pathogenesis should be explored in patients on chronic antiepileptic therapy with VPA if they have an apparently unexplained hypoglycemia
Corresponding author Eugenia Rota, M.D.

Department of Neurology, ASLAL San Giacomo Hospital Via E. Raggio 12, 15067,

Novi Ligure, Alessandria, Italy Tel. +39 0143-332431; Fax +39 0523-332576 Email: eugenia.rota.md@gmail.com

Received: 5 October 2020 Accepted: 3 February 2021 Published: 23 April 2021 
factor, and intracellular signaling proteins in the mechanism of action of VPA [2,3]. It has also recently emerged that VPA inhibits histone deacetylation and regulates the transcription of various genes. This effect implies it has a potential role as anti-cancer drug and as possible antidiabetic agent [4].

However, it has been demonstrated that VPA administration may cause numerous side effects and/or pharmacological interference and, thus, requires strict clinical and laboratory control, with serum level monitoring [5]. Indeed, VPA can lead to hyponatremia due to an inappropriate antidiuretic hormone secretion. It can also cause hematologic toxicity (thrombocytopenia, leucopenia, macrocytic anemia), severe liver damage, kidney failure, hypothyroidism, hyperammonemia, rhabdomyolysis, hyperandrogenism, pancreatitis, as well as teratogenicity. The mechanisms underlying most of these side effects have not yet been completely understood $[3,6,7]$.

It has been reported that drug-induced hypoglycemia as a cause of acute medical admissions ranges from $0.1 \%$ to $1.7 \%$ [8]. Literature reports several types of non-diabetic drugs that may induce hypoglycemia, including: NSAIDs, analgesics, antibacterials, antimalarials, pentamidine, $B$-adrenergic receptor antagonists (ß-blockers), antiarrhythmics, $\mathrm{ACE}$-inhibitors, fibrates, and antidepressants [9]. Ben Salem et al. also reported that some antiepileptic drugs (gabapentin, phentoin, topiramate) caused iatrogenic hypoglycemia [9]. It was hypothesized that the underlying mechanisms were insulin release or the reduction of its clearance, and/or an interference in the glucose metabolism [9].

Herein, we report a case of persistent hypoglycemia episodes, which were most likely induced by VPA, administered at an appropriate dosage in an epileptic patient.

\section{CASE PRESENTATION}

A 49-year-old male, affected by an epileptic syndrome from the birth, was admitted to our Neurology Unit for recurrent focal seizures to bilateral tonic-clonic seizures. His clinical history included diabetes mellitus (DM) type 2 treated by metformin, oligophrenia, associated with the epileptic syndrome, which was probably structural, related to perinatal hypoxic damage, and secondary psychosis, treated with risperidone.
He had been on VPA therapy, at $1000 \mathrm{mg}$ bid, for about a year before being hospitalized in our department. Two EEGs performed in the previous 2 months had shown no evidence of epilepsy.

The patient had been admitted to our Emergency Department the previous month for respiratory failure and bilateral pneumonia, which had evolved into septic shock requiring Intensive Care. Although recurrent hypoglycemia was observed, it was attributed both to the septic state and his reduced food intake.

His hepatic function was normal, i.e., alanine transaminase $30 \mathrm{mg} / \mathrm{dl}$, aspartate transaminase $35 \mathrm{mg} / \mathrm{dl}$, and ammonium levels were within normal levels $(35 \mathrm{mg} / \mathrm{dl})$, as was the renal function.

When he arrived in our Division, he was still on maintenance therapy, i.e., methylprednisolone (40 $\mathrm{mg}$ daily), prescribed when he had been admitted to the ICU, and risperidone $1 \mathrm{mg}$ daily: no variation was made to the treatment. He had continuous glucose infusions.

The patient was transferred to an Internal Medicine Department after a week, where the hypoglycemia episodes persisted and a generalized epileptic seizure occurred, even though his VPA plasma levels were in the therapeutic range.

A more in-depth medical history brought to light two previous diabetology consultations for hypoglycemia in the previous year. As the first hypothesis was that his symptoms were attributable to metformin, it was discontinued. Clinical investigations were made, firstly a diabetology assessment, dosing of C-peptide (it was within normal limits), and an abdomen CT scan.

The simultaneous determination of insulinemia and blood glucose, the insulinblood glucose ratio and the concomitant determination of insulinemia and blood glucose during 24-48-72 hours of fasting were unremarkable.

No neuroendocrine tumor was found by the abdomen $\mathrm{CT}$ scan, and neuron-specific enolase and chromogranin A were normal.

An iatrogenic effect was then hypothesized, with the suspicion that VPA played some kind of role. Therefore, the patient was transferred back to the Neurology Unit for rapid VPA withdrawal (500 $\mathrm{mg}$ bid for two days, then withdrawn), switching to levetiracetam (LVT) at $1000 \mathrm{mg}$ bid.

The hypoglycemic episodes persisted for another week, especially during the morning. 
It took 7 days for the blood glucose values to normalize after VPA withdrawal (Figure 1). A steady normoglycemic state was then maintained and epilepsy was kept under good control by levetiracetam.

\section{DISCUSSION}

VPA is known to have several side effects, including teratogenicity and hyperammonemia, with possible serious encephalopathy $[3,6]$ and it is difficult to predict the individual response to treatment and the appearance of side effects.

The recent developments of pharmacogenomics has brought to light the fact that there are numerous genes which may influence VPA metabolism, efficacy and safety. This may, in part, explain the individual variability of plasma levels and the onset of some serious side effects, including liver toxicity and teratogenicity [7].

Our patient had type $2 \mathrm{DM}$ and a good metabolic compensation. He was hospitalized for sepsis associated with respiratory failure and admitted to the ICU, where the emerging episodic hypoglycemia was attributed to his septic state. But the hypoglycemia progressed, despite the fact that the inflammatory state remitted. A diagnostic work up investigated into the possible pathogenesis of the hypoglycemia and reasonably ruled out: an iatrogenic nature (metformin), organic endogenous causes (insulin, congeni- tal metabolic diseases, and extra-pancreatic cancers), as well as functional endogenous causes (postprandial reactive hypoglycemia, pituitary and cortico-surrenalic insufficiency, liver disease, malnutrition), and a VPA hepatotoxicity syndrome.

As the C-peptide connects the insulin $\mathrm{A}$-chain to its $\mathrm{B}$-chain in the proinsulin molecule, it was dosed. Proinsulin is the precursor of the insulin synthesized in the ß-pancreatic cells. As C-peptide and insulin are released into the circulation in equimolar quantities by pancreas ß-cells, assessing circulating $\mathrm{C}$-peptide levels provides an indirect evaluation of insular $ß$ activity. Cpeptide assessment is often preferred to insulin assessment as it is metabolized in the proximal renal tubes and not by the liver and has a slower metabolism and lacks crossreactivity with antibodies directed towards insulin [10]. In our patient, the C-peptide tuned out to within the normal limits.

Moreover, the VPA level decrease observed after withdrawal was followed by a blood glucose increase (Figure 1), supporting the hypothesis that VPA played a direct role in hypoglycemia as a side effect, even though the decline in VPA serum concentrations took somewhat longer than expected.

This time course of VPA level decrease and glucose rise, after a week interval from the VPA nadir, pointed to a possible role of other factors, mainly pharmacological (the other drugs co-administered), but also clinical (the comorbidities), which might have af-
Figure 1. Glucose and Valproic Acid level time course (on the abscissa the days from admission to the Neurology Unit) 
fected the VPA half-life and the restoration of a normal glucose metabolism.

However, a VPA hepatotoxicity syndrome was ruled out by the normal liver function, including blood ammonia levels, and by the absence of jaundice.

The relationship between VPA and hypoglycemia has been reported above all in animal models.

Experiments in diabetic rats treated with VPA showed a dose-dependent reduction in blood glucose, which peaked around the $21^{\text {st }}$ day of administration (a reduction of $52.79 \%$ ), whilst in normal glycemic rats the peak was reached over the same period at a percentage of $15.63 \%$ [11]. These results and further findings on animal models [12] suggest that VPA has antidiabetic properties.

Moreover, literature has described some cases of neonatal hypoglycemia after in utero exposure to valproate [13-15].

Cases of VPA-induced hypoglycemia in adults have been reported almost exclusively in the context of hepatic failure due to VPA intoxication [16]. To the best of our knowledge, there are very few reports on hypoglycemia induced by therapeutical levels of VPA [17]. VPA has not been included in the list of non-diabetic agents reported as being responsible for hypoglycemia [18].

Apart from hepatic failure, it may be hypothesized that there are some other underlying mechanisms that mediate the hypoglycemic effect of VPA, i.e., insulin release, or its reduced clearance and/or interference with the glucose metabolism at multiple levels [9]. Indeed, on the basis that VPA may induce pancreatitis, it may be supposed that VPA has a metabolic influence on insulin release at a pancreatic level [9]. Moreover, it has been hypothesized that VPA acts as a substrate for the fatty acid $\beta$-oxidation pathway, leading to carnitine consumption. Therefore, when fatty acid $\beta$-oxidation is inhibited by a low carnitine level, the fasting hepatic gluconeogenesis may be decreased, leading to hypoglycemia [17].

\section{CONCLUSIONS}

Antiepileptic-induced iatrogenic hypoglycemia, in particular VPA-induced, has not been commonly reported, nor is it included in the side effects described in the VPA datasheet.

The case herein reported in an adult patient supports the hypothesis that VPA may induce hypoglycemia, by mechanisms and metabolic effects that remain to be defined, even at a therapeutical dosage.

Therefore, we strongly suggest that whenever there is a case of apparently unexplained hypoglycemia in a patient on chronic therapy with VPA, the possibility of there being an iatrogenic pathogenesis should be carefully evaluated.

As currently only anecdotal reports are available, the effect of VPA on glucose metabolism should be better clarified by future investigations. Indeed, a better understanding of these mechanisms may well lead to better and safer management of VPA therapy and may even prompt research on the possible, novel indications for metabolic control in patients with glucose intolerance.

Keypoints

- VPA may induce hypoglycemia, even at a therapeutical dosage.

- Cases of apparently unexplained hypoglycemia in patients on chronic VPA therapy should be carefully evaluated to rule out an iatrogenic pathogenesis.

- Hopefully, future studies will shed light on the mechanisms underlying the hypoglycemia induced by VPA and the effect VPA has on the glucose metabolism.

Consent to publication

The consent to publication was obtained from the patient's relative.

Funding

This article has been published without the support of sponsors.

Conflicts of interests

The authors declare they have no competing financial interests concerning the topics of this article. 


\section{REFERENCES}

1. Owens MJ, Nemeroff CB. Pharmacology of valproate. Psychopharmacol Bull 2003; 37 Suppl 2: $17-24$

2. Beghi E. Treating epilepsy across its different stages. Ther Adv Neurol Disord 2010; 3: 85-92; https://doi.org/10.1177/1756285609351945

3. Johannessen CU, Johannessen SI. Valproate: past, present, and future. CNS Drug Rev 2003; 9: 199-216; https://doi.org/10.1111/j.1527-3458.2003.tb00249.x

4. Yamato E. High dose of histone deacetylase inhibitors affects insulin secretory mechanism of pancreatic beta cell line. Endocr Regul 2018; 52: 21-6; https://doi.org/10.2478/enr-2018-0004

5. Dreifuss FE, Langer DH. Side effects of valproate. Am J Med 1988; 84 (1A): 34-41; https:// doi.org/10.1016/0002-9343(88)90055-1

6. Chopra A, Kolla BP, Mansukhani MP, et al. Valproate-induced hyperammonemic encephalopathy: an update on risk factors, clinical correlates and management. Gen Hosp Psychiatry 2012; 34: 290-8; https://doi.org/10.1016/j.genhosppsych.2011.12.009

7. Zhu MM, Li HL, Shi LH, et al. The pharmacogenomics of valproic acid.J Hum Genet 2017; 62: 1009-14; https://doi.org/10.1038/jhg.2017.91

8. Chan TY, Lee KK, Chan AW, et al. Utilization of antidiabetic drugs in Hong Kong: relation to the common occurrence of antidiabetic drug-induced hypoglycemia amongst acute medical admissions and the relative prevalence of NIDDM. Int J Clin Pharmacol Ther 1996; 34: 43-6

9. Ben Salem C, Fathallah N, Hmouda H, et al. Drug-induced hypoglycaemia: an update. Drug Saf 2011; 34: 21-45; https://doi.org/10.2165/11538290-000000000-00000

10. Jones AG, Hattersley AT. The clinical utility of $\mathrm{C}$-peptide measurement in the care of patients with diabetes. Diabet Med 2013; 30: 803-17; https://doi.org/10.1111/dme.12159

11. Rabadiya S, Bhadada S, Dudhrejiya A, et al. Magnesium valproate ameliorates type 1 diabetes and cardiomyopathy in diabetic rats through estrogen receptors. Biomed Pharmacother 2018; 97: 919-27; https://doi.org/10.1016/j.biopha.2017.10.137

12. Akindele AJ, Otuguor E, Singh D, et al. Hypoglycemic, antilipidemic and antioxidant effects of valproic acid in alloxan-induced diabetic rats. Eur J Pharmacol 2015; 5: 174-83; https://doi. org/10.1016/j.ejphar.2015.05.044

13. Ebbesen F, Joergensen A, Hoseth E, et al. Neonatal hypoglycaemia and withdrawal symptoms after exposure in utero to valproate. Arch Dis Child Fetal Neonatal Ed 2000; 83: F124-F129; https://doi.org/10.1136/fn.83.2.F124

14. Çoban D, Kurtoğlu S, Akın MA, et al. Neonatal episodic hypoglycemia: a finding of valproic acid withdrawal. J Clin Res Pediatr Endocrinol 2010; 2: 92-4; https://doi.org/10.4274/jcrpe.v2i2.92

15. Bruel H, Chabrolle JP, Amusini P, et al. Hyperammonia, hypoglycemia and thrombocytopenia in a newborn after materanl treatment with valproate. Arch Pediatr 2001; 8: 446-7; https://doi. org/10.1016/S0929-693X(00)00223-2

16. van den Broek MP, Sikma MA, Ververs TF, et al. Severe valproic acid intoxication: case study on the unbound fraction and the applicability of extracorporeal elimination. Eur J Emerg Med 2009; 16: 330-2; https://doi.org/10.1097/MEJ.0b013e32832c7b18

17. Sai Naveen S, Siva Anoop Y. Sodium Valproate Induced Hypoglycemia: A Rare Case Report. International Research Journal of Pharmacy and Medical Sciences (IRJPMS) 2019; 2: 48-9

18. Murad MH, Coto-Yglesias F, Wang AT, et al. Drug induced hypoglycemia: a systematic review. J Clin Endocrinol Metab 2009; 94: 741-5; https://doi.org/10.1210/jc.2008-1416 